\title{
Monte Carlo simulation tool for online treatment monitoring in hadrontherapy with in-beam PET: a patient study
}

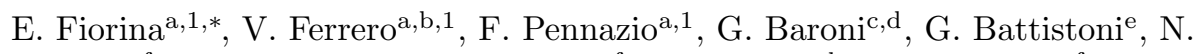 \\ Belcari $^{\mathrm{f}, \mathrm{g}}$, P. Cerello ${ }^{\mathrm{a}}$, N. Camarlinghi ${ }^{\mathrm{f}, \mathrm{g}}$, M. Ciocca $^{\mathrm{d}}$, A. Del Guerra ${ }^{\mathrm{f}, \mathrm{g}}$, M. \\ Donetti $^{\mathrm{d}}$, A. Ferrari $^{\mathrm{h}}$, S. Giordanengo ${ }^{\mathrm{a}}$, G. Giraudo ${ }^{\mathrm{a}}$, A. Mairani $^{\mathrm{d}}$, M. \\ Morrocchi $^{\mathrm{f}, \mathrm{g}}$, C. Peroni ${ }^{\mathrm{a}, \mathrm{b}}$, A. Rivetti ${ }^{\mathrm{a}}$, M.D. Da Rocha Rolo ${ }^{\mathrm{a}}$, S. Rossi ${ }^{\mathrm{d}}$, V.

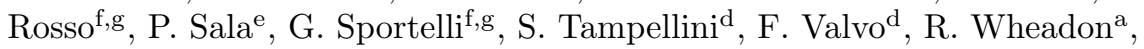 \\ M.G. Bisogni ${ }^{\mathrm{f}, \mathrm{g}}$ \\ ${ }^{a}$ Istituto Nazionale di Fisica Nucleare, sez. Torino, via Giuria 1, 10125 Torino, Italy \\ ${ }^{b}$ Università degli Studi di Torino, dipartimento di Fisica, via Giuria 1, 10125 Torino, Italy \\ ${ }^{c}$ Politecnico di Milano, piazza L. Da Vinci 32, 20133 Milano, Italy \\ ${ }^{d}$ Fondazione CNAO, Centro Nazionale di Adroterapia Oncologica, strada Campeggi 53, \\ 27100 Pavia, Italy \\ ${ }^{e}$ Istituto Nazionale di Fisica Nucleare, sez. Milano, via Celoria 16, 20133 Milano, Italy \\ ${ }^{f}$ Università di Pisa, dipartimento di Fisica, lungarno A. Pacinotti 43, 56126 Pisa, Italy \\ ${ }^{g}$ Istituto Nazionale di Fisica Nucleare, sez. Pisa, largo B. Pontecorvo 3, 56127 Pisa, Italy \\ ${ }^{h}$ CERN, CH-1211, Geneva 23, Switzerland
}

\begin{abstract}
Hadrontherapy is a method for treating cancer with very targeted dose distributions and enhanced radiobiological effects. To fully exploit these advantages, in vivo range monitoring systems are required. These devices measure, preferably during the treatment, the secondary radiation generated by the beam-tissue interactions. However, since correlation of the secondary radiation distribution with the dose is not straightforward, Monte Carlo (MC) simulations are very important for treatment quality assessment.

The INSIDE project constructed an in-beam PET scanner to detect signals generated by the positron-emitting isotopes resulting from projectile-target fragmentation. In addition, a FLUKA-based simulation tool was developed to predict the corresponding reference PET images using a detailed scanner model.

The INSIDE in-beam PET was used to monitor two consecutive proton treatment sessions on a patient at the Italian Center for Oncological Hadrontherapy (CNAO). The reconstructed PET images were updated every 10 seconds providing a near real-time quality assessment. By half-way through the treatment, the statistics of the measured PET images were already significant enough to be compared with the simulations with average differences in the activity range
\end{abstract}

\footnotetext{
* Corresponding author

Email address: fiorina@to.infn.it (E. Fiorina)

${ }^{1}$ E. Fiorina, V. Ferrero and F. Pennazio equally contributed to this work.
} 
less than $2.5 \mathrm{~mm}$ along the beam direction. Without taking into account any preferential direction, differences within $1 \mathrm{~mm}$ were found.

In this paper, the INSIDE MC simulation tool is described and the results of the first in-vivo agreement evaluation are reported. These results have justified a clinical trial, in which the MC simulation tool will be used on a daily basis to study the compliance tolerances between the measured and simulated PET images.

Keywords: hadrontherapy, in-beam PET, range monitoring, Monte Carlo simulation

2010 MSC: 00-01, 99-00

\section{Introduction}

\subsection{Treatment monitoring in hadrontherapy}

Treatment monitoring in hadrontherapy is a vital tool for maximizing the benefits from the increased conformation of the released dose to the tumour volume when compared with conventional photon radiotherapy [1]. This increased conformation is due to the fact that almost all the energy of the charged particles is deposited where they stop, with steep gradients in the Bragg Peak region, and therefore confirmation of the particle range by measurement can help to increase the effectiveness of the treatment and minimize collateral damage to healthy tissues.

There are some sources of uncertainty in the determination of the actual particle range, such as approximations in dose calculation and Hounsfield Units conversion, mis-positioning and variations of patient anatomy.

Furthermore, several studies have already explored the impact of uncertainties in models onto the treatment planning optimization. For example, the biological model uncertainties and their impact on a treatment plan optimization based on a variable Relative Biological Effectiveness (RBE) are discussed in [2]; the treatment planning improvement by using an original parametrization of the lateral dose profiles is reported in [3]; the use of ion transmission imaging in order to provide valuable information on the patient-specific stopping properties is explained in [4].

To avoid critical complications, physicians must design robust treatment plans allowing for small variations in particle range, applying $(2.5-3.5) \%+(1-$ 3) $\mathrm{mm}$ safety margins to the tumour volume [5]. In addition, if anatomical changes are expected during the course of the treatment then further Computed Tomography (CT) scans must also be planned. A monitoring device, able to evaluate the compliance of the delivered treatment with the prescription, would be very useful in cancers with an early response to the therapy (i.e., tumour volume reduction) or in districts traversed by the beam where tissues are prone to physiological or radio-induced variations in density (e.g. intestine, paranasal sinuses) 6] 7]. Access to this information could be a first step towards new optimizations in treatment planning and adaptive therapy [1]. 


\begin{tabular}{|c|c|c|c|}
\hline Isotope & $\mathrm{Z}$ & $\mathrm{A}$ & Half life \\
\hline $\mathrm{C}$ & 6 & 11 & $1220.04 \mathrm{~s}$ \\
& & 10 & $19.29 \mathrm{~s}$ \\
\hline $\mathrm{O}$ & 8 & 15 & $122.24 \mathrm{~s}$ \\
& & 14 & $70.6 \mathrm{~s}$ \\
& & 13 & $8.58 \mathrm{~ms}$ \\
\hline $\mathrm{N}$ & 7 & 13 & $597.9 \mathrm{~s}$ \\
& & 12 & $11 . \mathrm{ms}$ \\
\hline $\mathrm{B}$ & 5 & 8 & $770 . \mathrm{ms}$ \\
\hline $\mathrm{K}$ & 19 & 38 & $458.2 \mathrm{~s}$ \\
\hline $\mathrm{P}$ & 14 & 30 & $149.89 \mathrm{~s}$ \\
\hline $\mathrm{Cl}$ & 17 & 34 & $1.52 \mathrm{~s}$ \\
\hline
\end{tabular}

Table 1: Main positron-emitting isotopes produced during hadrontherapy treatments.

Several research groups have already built or have under construction detection systems able to determine the particle range inside the patient body by means of secondary radiation 8 , 9]. The main signals generated from the beam/patient tissue interactions are: prompt photons obtained from nuclear de-excitation, secondary charged particles generated by nuclear fragmentation, and photons at $511 \mathrm{keV}$ emitted back-to-back by positron annihilations.

The first two signals are promptly emitted during irradiation, with a spatial and temporal structure that is strongly correlated with the beam current irradiation. Moreover, their distributions depend on the target elemental composition, beam energy and detection angle 10 11.

On the other side, the PET signal is asynchronous because it is generated by positron-emitting isotopes coming from nuclei fragmentation. Oxygen and carbon isotopes are the most abundant and their half-life is of the order of minutes and seconds, as shown in Table 1 . Some isotopes have a shorter half-life $\left({ }^{12} N\right.$, ${ }^{8} \mathrm{~B},{ }^{13} \mathrm{O}$ ) and, even if they are not the main contributions, can be used as a synchronous signal with minimum delay with respect to the treatment delivery [12.

Positron Emission Tomography (PET) is a well-established in-vivo imaging technique and the most mature method for range monitoring in hadrontherapy in clinics 13. There are three operational modalities that have been investigated to make this range monitoring approach available in clinics: off-line, in-room and in-beam PET [14.

Off-line PET uses a commercial full-ring scanner sited nearby the treatment room. Commonly, an integrated CT system is available, useful for image coregistration. In this way, the PET images have a high resolution and the clinical workflow is not slowed down because, after the irradiation, the patient is immediately moved away, as in the normal clinical routine. The main limitations of this approach are due to the biological wash-out of the positron emitters and their relatively short decay times, similar to the time needed to reposition the patient in the $\mathrm{PET} / \mathrm{CT}$ room. The time required for image acquisition can be 
up to 30 minutes [15.

In-room PET commonly uses a stand-alone full-ring PET scanner positioned in the treatment room in order to minimize the biological wash-out and the signal degradation. Sometimes, the same treatment table is also used, minimizing any possible issues related to patient repositioning. This technique has a higher sensitivity compared to the off-line approach and the PET image acquisition time can be reduced (about $5 \mathrm{~min}$ ) [16. However, this approach slows down the clinical workflow. In addition, it requires the installation of a PET scanner in the treatment room and the development of a dedicated image co-registration procedure if an additional CT scan is not acquired.

In-beam PET is based on a custom detector, able to acquire data during irradiation of the patient. Some geometrical constraints must be addressed for compatibility with the beam line and the clinical procedures. In many cases, a dual-head geometry is chosen [17] [18] 19] and, moreover, a good agreement with MC predictions has been demonstrated on phantoms (e.g. in 20]). The in-beam PET approach permits the detection, at least in principle, of all the signals coming from positron-emitting isotopes and minimizes the contribution of the biological wash-out. An online monitoring device able to acquire and analyse data during the treatment is becoming a really desired tool in clinics [1]. Such an in-beam PET device, thanks to technological improvements, is now possible.

In this scenario, the INSIDE (INnovative Solution for In-beam Dosimetry in hadronthErapy) project was created with the goal of building a bi-modal system to perform online monitoring in hadrontherapy 21] 22, 23] 24]. The system is composed of an in-beam Positron Emission Tomography (PET) scanner and a secondary charged particle tracker, called Dose Profiler. The INSIDE system uses state-of-the-art technology Silicon Photomultipliers, exploiting their compactness and timing features, in order to build a system compatible with the geometry constraints of the treatment rooms with horizontal beam lines at the Italian Centre for Oncological Hadrontherapy (CNAO) synchrotron facility in Pavia, Italy.

The INSIDE Dose Profiler, completed at the beginning of 2017, is still under calibration and validation [25] and will not be presented in this work.

\subsection{The INSIDE in-beam PET system}

Assembly of the INSIDE in-beam PET scanner was completed in January 2016 and subsequently installed at the CNAO synchrotron facility [21]. After the commissioning phase [26] 27] 28, in December 2016, two patient treatment sessions were monitored by the system, which demonstrated the capability of providing a reliable response after about half of the treatment irradiation and with a $6 s$ delay with respect to beam delivery (i.e., the result of the on-the-fly analysis refers to data collected up to $6 s$ before) 29 .

The INSIDE in-beam PET scanner is mounted on a mobile support and features two panels of photo-detectors inside two boxes placed above and under the patient bed as shown in Fig 1. The distance between the two PET panels was set to $60 \mathrm{~cm}$, in order to preserve the patient's safety and comfort. 


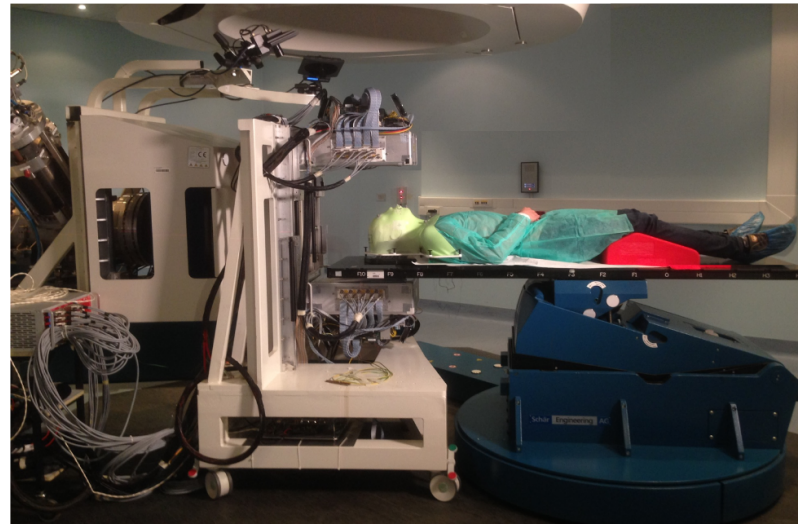

Figure 1: Enactment of the first patient test with the INSIDE in-beam PET scanner. The system is installed in one of the CNAO treatment rooms at the working position.

Each PET panel is composed of $5 \times 2$ modules of Luthetium Fine Silicate (LFS) pixelated scintillating crystals coupled one-to-one to Multi Pixel Photon Counters (MPPCs) by Hamamatsu ${ }^{2}(3.2 \mathrm{~mm}$ pitch). The front-end electronics mounted inside the two PET boxes is based on the TOFPET ASIC developed in the framework of the EndoTOFPET project [30]. Data is filtered in energy and sent to a Data Acquisition (DAQ) software thanks to one Field-Programmable Gate Array (FPGA) board for each detection module. The DAQ software is able to find coincidences, reconstruct the PET image at any given acquisition time with a Maximum Likelihood Expectation Maximization (MLEM) algorithm $\left(F O V=11.2 \times 22.4 \times 26.4 \mathrm{~cm}^{3}\right.$, voxel size $\left.=1.6 \times 1.6 \times 1.6 \mathrm{~mm}^{3}\right)$, analyse the $4 \mathrm{D}$ PET image and return the result of the evaluation with a delay of a few seconds with respect to the beam delivery.

With the mechanical design of the current support, the INSIDE in-beam PET is only compatible with some treatments in the head-neck region geometry. A particularly feasible geometry is the cranio-caudal field, where the irradiation field is scanned by the CNAO horizontal beam line and the primaries impinge on the top of the patient head.

\subsection{Monte Carlo simulations}

Monte Carlo simulations are extensively used in modern cancer treatment, mainly to calculate the dose distribution and validate the treatment plan. Noninvasive in-vivo monitoring solutions based on secondary particle detection require prior information from $\mathrm{MC}$ tools to be able to assess the compliance of the primary particle range inside the patient with the prescription [31] 32].

The production of secondary radiation involves nuclear interactions between the primary beam and human tissues. At the energies that are relevant in

\footnotetext{
${ }^{2}$ http://www.hamamatsu.com
} 
the hadrontherapy field, nuclear interactions and fragmentations are not yet completely rigorously modelled. The current status of their implementation in MC codes is reviewed in [33. Hopefully, the lack of experimental informations about fragmentation cross sections will be overcome in the next years by the results of several ongoing projects (e.g., 34, 35]).

In any case, even if the reliability of Monte Carlo simulations could be improved, they are still the most successful and effective way to compare the secondary particle signal with the treatment prescription. In fact, the secondary particle production is not straightforwardly correlated with data commonly available from the Treatment Planning System (TPS), such as the dose distribution, and therefore more reference information is needed [36].

The MC simulation technique was already successfully used in other works (among them [37, 38, 39, 40]) for the evaluation of experimental PET images, acquired on patients with in-room or after treatment PET scanners. In Section 7. the results reported in this paper will be compared with some of these other studies.

\section{Material and Methods}

\subsection{The INSIDE MC simulation tool}

The INSIDE collaboration developed a MC simulation tool for predicting the expected 4D PET image based on the individual clinical situation.

This tool was developed in the FLUKA framework [41] 42] [43] [44].

To obtain a simulated 4D PET image fully comparable with the measurements, the following elements are thoroughly characterized and modelled:

- the CNAO beam line

- the primary particle beam

- the patient morphology and positioning

- the treatment plan

- the primary beam temporal structure

- the INSIDE in-beam PET scanner

In Fig. 2 the implemented geometry is shown. The patient position during irradiation is supine, head first.

CNAO beam line. The geometry and materials of the CNAO horizontal beam line of the treatment room nr. 3 were reproduced as reported in [45, [46.

All the inserts along the beam path are taken into account: in particular, the monitor chambers of the Dose Delivery System (DDS) [47 and the ripple filters in case of carbon ions. 


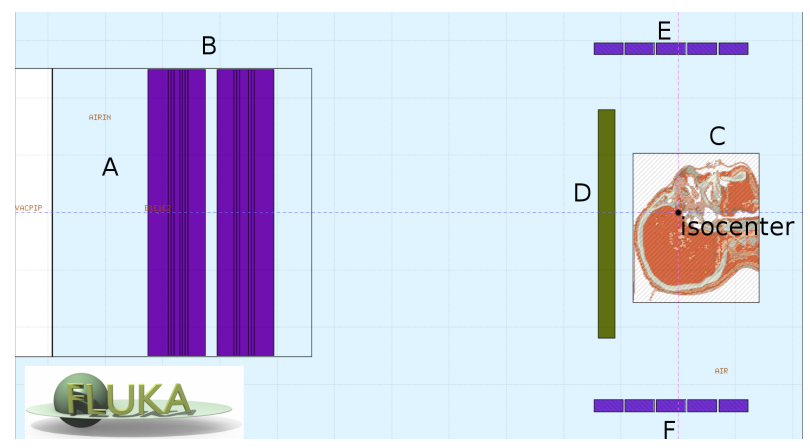

Figure 2: Geometry implemented in the Monte Carlo simulation tool in FLUKA. A) CNAO beam line. B) Monitor chambers of the Dose Delivery System. C) Patient CT. D) Range shifter, $3 \mathrm{~cm}$ water equivalent thick. E) INSIDE in-beam PET panel (above patient). F) INSIDE in-beam PET panel (below patient).

Primary particle beam. The dimension of the proton beam was modelled to reproduce in the simulation the trend found in the measurements reported in [48. In [48, the Full Width Half Maximum $(F W H M \mathrm{~cm})$ of a proton beam in air at the isocenter as a function of the beam energy $(E[\mathrm{MeV}])$ was compatible with the following model:

$$
F W H M=\frac{A}{E-B}+C
$$

where $A=70.50 \pm 2.47, B=25.51 \pm 1.30$ and $C=0.39 \pm 0.01$.

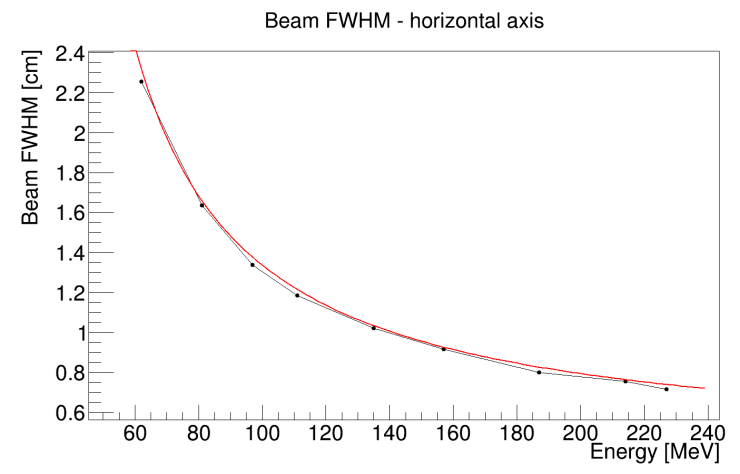

Figure 3: Proton beam FWHM at the isocenter in air: comparison between MC simulation (black points) and the model calculated starting from experimental measurements as reported in [4] (red line).

In Fig 3 the experimental model for proton beams is compared with the points obtained with the INSIDE MC simulation tool in the same measurement conditions (in air, at the isocenter).

The agreement between the experimental and simulated beam dimension is within $1 \mathrm{~mm}$ and was obtained by simulating a proton beam with a FWHM in 
$\mathrm{cm}$ that changes as a function of the beam energy $E$ in $M e V$ as follow:

$$
F W H M=p_{5} * E^{5}+p_{4} * E^{4}+p_{3} * E^{3}+p_{2} * E^{2}+p_{1} * E+p_{0}
$$

where $p_{5}=(-8.63 \pm 6.85) 10^{-11}\left[\mathrm{~cm} / \mathrm{MeV}^{5}\right], p_{4}=(7.02 \pm 4.94) 10^{-8}\left[\mathrm{~cm} / \mathrm{MeV}^{4}\right]$, $p_{3}=(-2.26 \pm 1.37) 10^{-5}\left[\mathrm{~cm} / \mathrm{MeV}^{3}\right], p_{2}=(3.61 \pm 1.82) 10^{-3}\left[\mathrm{~cm} / \mathrm{MeV}^{2}\right], p_{1}=$ $(-2.87 \pm 1.15) 10^{-1}[\mathrm{~cm} / \mathrm{MeV}]$ and $p_{0}=9.48 \pm 2.73[\mathrm{~cm}]$.

In 49 a similar approach was used to determine the lateral dose spread at the Heidelberg Ion-Beam Therapy Centre (HIT) synchrotron facility in Germany.

Patient morphology and positioning. The patient was simulated by using the CT scan on which the treatment plan was calculated. Human tissues are simulated using the materials and Hounsfield Units (HU) conversion curves reported in [50, which are already implemented in FLUKA and commonly used in clinics [41.

The patient positioning was simulated in agreement with the corresponding DICOM tags so as to place the isocenter of the treatment room at the same position as the experimental setup. Any other additional inserts positioned on the beam path (such as the range shifter shown in Fig. 22) are thoroughly simulated as physical objects.

Treatment plan. The treatment plan summarizes all the physical quantities that impact on the prescribed dose distribution.

At the CNAO centre, the patient treatment plans are calculated by means of a Syngo PT Planning Treatment Planning System (TPS) (version VB10, Siemens) [51.

In order to carefully simulate the experimental irradiation pattern in the spatial coordinates, the information contained in the treatment plan was included in the input file of the INSIDE MC simulation tool.

In particular, the particle type, the spot positions, the number of primaries per spot and the beam energies are taken into account to evaluate the spatial distribution of the generated positron-emitting isotopes.

Primary beam temporal structure. The reliability of an in-beam PET simulation depends on careful simulation of the event timing.

In fact, (1) the positron-emitter isotope production is strongly correlated to the temporal primary particle delivery and (2) the positron emission is determined by the corresponding isotope half-life.

PET image characteristics are fundamentally dependent on the acquisition time but with a non-trivial relationship that includes both the isotopes production time (synchronous with the irradiation) and the photon pairs detection time (asynchronous).

The CNAO synchrotron facility delivers the particle beam by alternating an acceleration phase (called inter-spill) and a delivery phase (in-spill) [52].

During the inter-spill phase, the charged particles are accelerated at the prescribed energy and no primary radiation is present inside the treatment room. Therefore, the asynchronous signal coming from positron annihilations 
has no prompt radiation background and can be successfully used to obtained in-beam PET images with good signal-to-noise ratio and negligible physiological washout.

During the in-spill phase, the INSIDE system acquires data but the signals coming from positron emitters are hidden among other prompt signals (pair production, prompt photon, neutrons, charged particles) and a filtering procedure is needed. In [26] an example of a filtering procedure implemented on data acquired with a small prototype of the INSIDE scanner has been reported. Moreover, a filtering procedure to remove prompt noise from in-spill data of the INSIDE PET scanner is under implementation.

The FLUKA simulation takes into account only the asynchronous signal coming from positron annihilation generated by the emitting isotopes. Although the short-lived isotopes contribution (half-life less than $1 s$ ) are also simulated, only inter-spill data was considered in the following analysis.

In the INSIDE MC simulation tool, the temporal structure of the beam delivery can be taken into account by using data provided by the DDS installed into the CNAO beam line [47. The number of delivered primary particles and the lateral beam position are sampled any $50 \mathrm{~ms}$ or whenever the irradiation point (i.e. spot) changes.

In Fig. 4, the agreement between simulated and measured coincidence event rates is shown: during the spills (corresponding to the peaks both in the simulated and experimental plots), the acquired coincidence rate is higher because of the unfiltered prompt signals, while only positron annihilation is considered in the simulation. During the inter-spill phase, the simulated and measured statistics are comparable. During the beam delivery, the coincidence event rate increases because of the continuous growing isotope production, while, when the treatment ends, the coincidence event rate starts decreasing because the isotope decays are no longer compensated by their production.

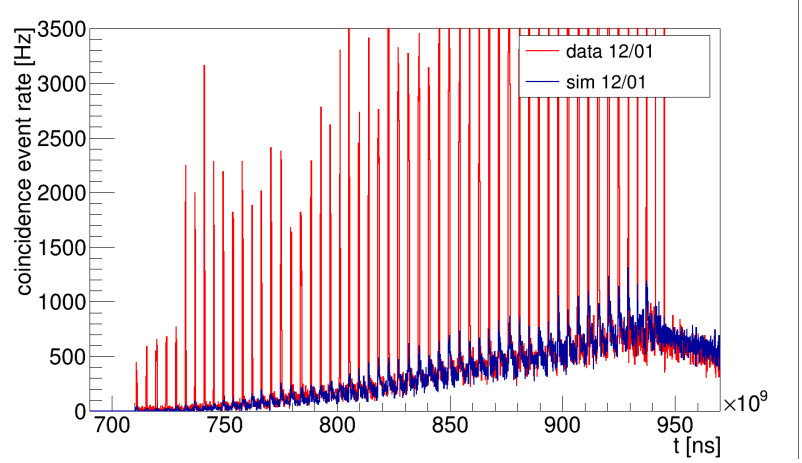

Figure 4: Coincidence event rate during the treatment of the first patient monitored by the INSIDE in-beam PET system (2016/12/01): data is shown in red, simulation in blue. Experimental in-spill peaks are out of scale to focus on the inter-spill contributions. 
INSIDE in-beam PET scanner. The detector is simulated by considering the materials and geometry of the scanner.

The energy and time resolutions obtained during the characterization phase of the INSIDE in-beam PET scanner with positron emitter sources for calibration are included. Further details about the calibration and characterization with phantoms are reported in 21] [53, 28, 27.

The simulated events are analysed using the same parameters as in the experimental data (coincidence time window of $2 \mathrm{~ns}$ and energy window of $0.1 \mathrm{MeV}$ to select the events in the photopeak region).

\section{The MC in-beam PET simulation workflow}

Because the positron-emitting isotopes give a statistically poor signal, all statistics must be simulated in order to obtain the most reliable 4D PET image to be compared with the experimental measure.

For reasons of computing time, the INSIDE in-beam PET simulation tool is based on a two-step simulation in FLUKA, separating the positron-emitting isotopes production in the first step (synchronous signal with respect to the primary delivery) and the positron annihilation in the second step (asynchronous signal).

In the first step, $1 / 100$ of the primaries is tracked in order to obtain a spatial and temporal distribution of the production of positron-emitter isotopes. In the second step, the full statistics are used and, on the basis of the spatial and temporal map of the isotopes, positrons are generated in agreement with the corresponding isotope half-life.

The energy deposition events in the detector are saved and processed to simulate the signal formation and acquisition chain. The events in coincidence are identified using the same procedure as that used for the experimental data.

At the end of the simulation, a time-tagged coincidence event list is generated as for the experimental acquisition, in order to build the 4D PET image to study the evolution of the positron-emitting isotopes signal in space and time. Presently, the comparison between the MC simulated images and the experimental ones is performed offline, although its on-the-fly implementation could be straightforwardly integrated in the near future.

\section{4D PET image analysis}

Preprocessing. A median filter with a $11.2 \times 11.2 \times 11.2 \mathrm{~mm}^{3}$ kernel $(7 \times 7 \times 7$ voxels) is applied to the $4 \mathrm{D}$ PET images in order to reduce the salt and pepper noise related to the poor statistics in the image, while preserving edges.

To mitigate the contribution of small intensity variations in the image not correlated with the real range difference to be detected, the simulated and experimental 4D PET images are equalized [54]. 
Pearson's Correlation Coefficient. The Pearson's Correlation Coefficient between two 3D PET images at the same time is calculated to evaluate the overall agreement in time between the two image series.

The Pearson's Correlation Coefficient (PCC) is:

$$
P C C=\frac{\sum_{i=1}^{n}\left(x_{i}-\bar{x}\right)\left(y_{i}-\bar{y}\right)}{\sqrt{\sum_{i=1}^{n}\left(x_{i}-\bar{x}\right)^{2}} \sqrt{\sum_{i=1}^{n}\left(y_{i}-\bar{y}\right)^{2}}}
$$

where $x_{i}\left(y_{i}\right)$ and $\bar{x}(\bar{y})$ are the intensity of the 3D PET image in the voxel $i$ and the average intensity value, respectively.

To achieve a reliable and effective range monitoring with a PET system, the most important Region Of Interest (ROI) in the 3D PET image to consider is the activity distal fall off, just before the Bragg Peak position [55. Therefore, for each activity profile in the beam direction, only the voxels between the first above the $95 \%$ of the maximum profile intensity and the last above $25 \%$ are considered in the PCC calculation. Hence, only the activity profiles that cross the irradiated volume can actually give a contribution to the ROI definition. Since in the two images to be compared the ROI must be the same, the logic union of the two ROI's calculated independently is considered.

Beam's Eye View analysis. Other studies [56, 38, 40, suggest to assess the range by considering the preferential direction of the beam. This type of approach is called Beam's Eye View $(B E V)$ and is designed to maximize the sensitivity in the detection of differences between the measured and prescribed range.

The implemented BEV analysis prescribes (1) the extraction of an isoactivity surface $S$ from each 3D PET image, (2) the activity range $R$ calculation for each activity profile crossing the irradiated volume, (3) the evaluation of metrics extracted from the $R$ distribution.

The 3D iso-activity surface is extracted by a sequence of threshold-erosiondilation filters. The intensity threshold is set at the $10 \%$ of the maximum activity in the 3D PET image. To avoid surface detection driven only by poor statistics or noise, only the largest surface in each is taken into account.

For all the activity profiles crossing the irradiating volume, the activity range $R$ is defined by evaluating the difference between the entrance and exit points in the surface $S$ in the beam direction.

To compare two 4D PET images, the distribution of the difference between the activity range from data $R_{\exp }$ and from simulation $R_{s i m}$ is taken into account. The average range difference and the FWHM of the range difference distribution will be reported in Section 6

The main drawback of the BEV method is the high sensitivity to detector mis-positioning, and, therefore, the method gains importance for systems with negligible uncertainty in the detector positioning. Unfortunately, at the time of the clinical test the INSIDE in-beam PET scanner did not have a dedicated positioning system yet, so an uncertainty of $2-3 \mathrm{~mm}$ related to the manual alignment of the scanner with the lasers of the treatment room has to be taken into account. 
Overall View analysis. To evaluate the agreement between two 4D PET images reducing the contribution of detector mis-positioning, the Overall View $(O V)$ analysis has been implemented.

The $3 \mathrm{D}$ iso-activity surface at the $10 \%$ of the maximum activity is extracted, as reported in the previous paragraph.

To compare two 4D PET images (i.e. experimental and simulated images), the distribution of the minimum Euclidean distance between the two iso-activity surfaces $\delta S$, calculated voxel-by-voxel, is considered, with no preferential direction in the analysis.

Furthermore, only the contributions included in the actually irradiated volume are taken into account. The average distance and the FWHM of the surface distance distribution are reported in Section 6 to evaluate the agreement between the 4D PET images.

The OV method was designed to be less sensitive to detector positioning uncertainty with respect to the BEV method. On the other hand, the OV method is also less sensitive to little and localized range variation in the irradiated volume.

\section{Clinical case study}

The first patient monitored with the INSIDE in-beam PET scanner was a male, 56 years old, affected by carcinoma of the lacrimal gland. The prescribed dose was equal to $60 G y(R B E)$ in 30 daily proton fractions (2 Gy (RBE) each). A fixed $R B E$ value of 1.1 was used for planning.

The treatment was delivered with an horizontal beam line, using two orthogonal non-coplanar irradiation fields (one right lateral and one cranio-caudal).

The INSIDE in-beam PET monitored the cranio-caudal field of the $28^{\text {th }}(2016 / 12 / 01)$ and $29^{t h}(2016 / 12 / 02)$ fractions.

The monitored irradiation field prescribed $3.710^{10}$ protons with energy in the $[66.3,144.4] \mathrm{MeV}$ interval. The treatment delivery time of each monitored field was $240 s$ and the INSIDE in-beam PET scanner acquired data from the beginning of the treatment up to $30 s$ after the end of the proton delivery. This additional time corresponded to the time needed to reach the treatment bed and remove the scanner.

The clinical study was performed in accordance with relevant guidelines and regulations and was approved by the local ethics committee (request number 20170011630); informed consent was obtained from the patient. These acquisitions were already analysed in [29] to thoroughly compare in time the images of the two treatment sessions.

\section{Results}

The dataset considered in this work is composed of three 4D PET images: data 12/01 labels the measurement of the first monitoring acquisition, data 12/02 is the subsequent day experimental 4D PET image and simulation 12/01 
is the result of the MC simulation performed by means of the INSIDE MC simulation tool developed in FLUKA.
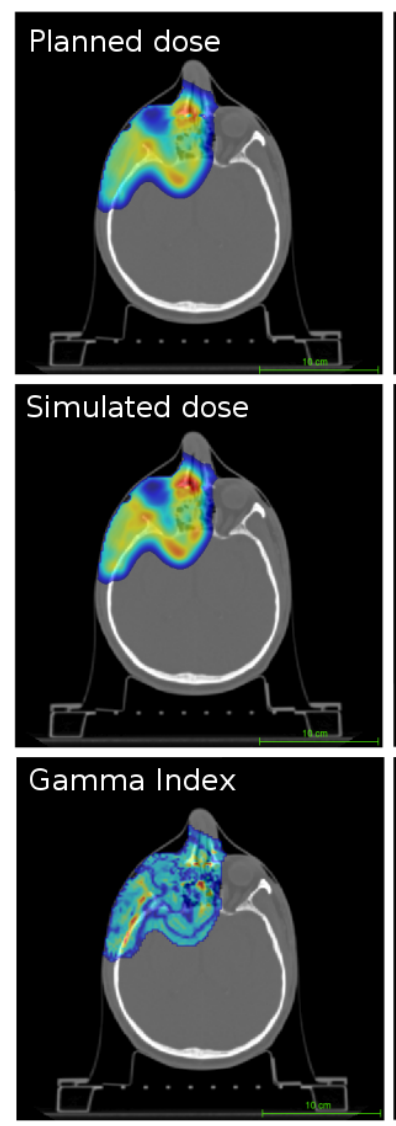
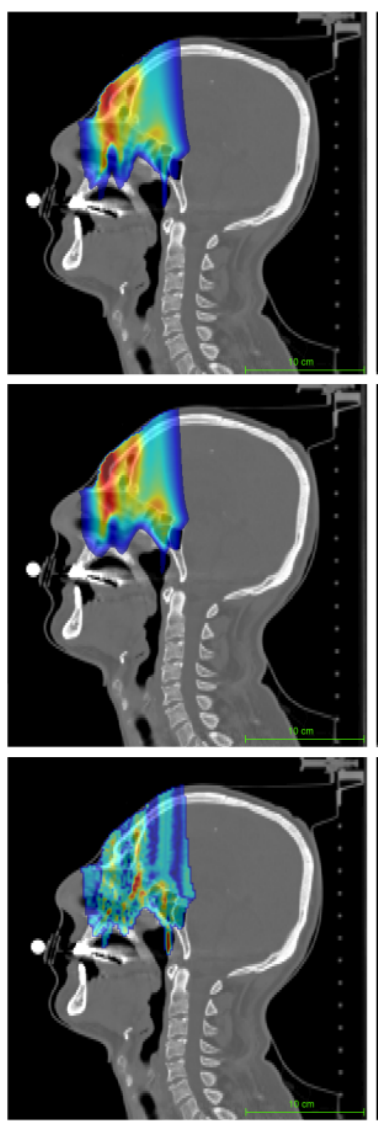
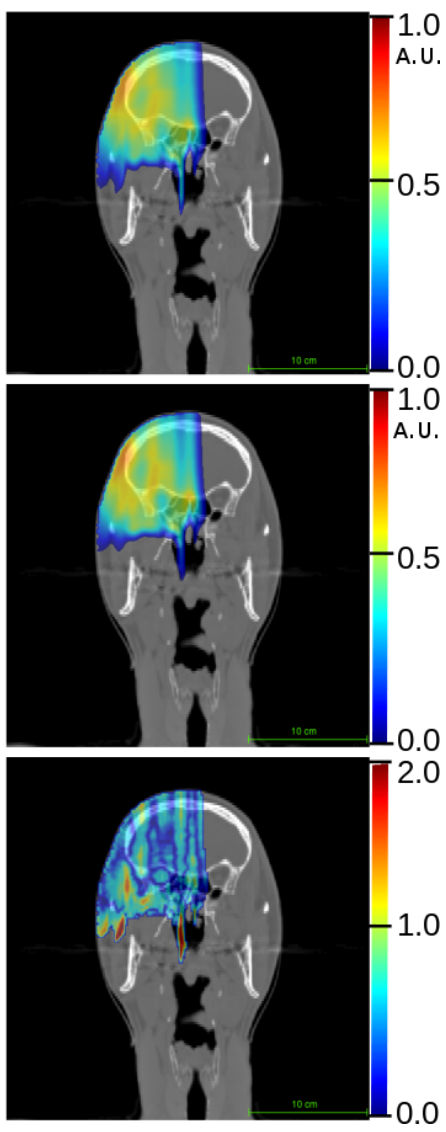

Figure 5: Comparison between the prescribed dose distribution (upper row), obtained from the RT Dose DICOM file, and the simulated dose distribution (central row) calculated by means of the INSIDE in-beam PET Monte Carlo simulation. The dose distributions are normalized to the maximum and overlaid in colour on the patient's CT. The Gamma Index calculated with the $3 \%-3 \mathrm{~mm}$ criteria is also shown (lower row).

Treatment simulation accuracy. To assess the overall accuracy in the simulation of the treatment, the prescribed and simulated dose are compared (Fig. 5). In order to evaluate the agreement between the two dose distributions, the Gamma Index was calculated as reported in [57.

In the Gamma Index evaluation, only voxels with a dose value above the $10 \%$ of the maximum and belonging to the patient body, according to CT segmentation, were taken into account. The passing rates of the Gamma Index between these two 3D images are $91 \%$ with $3 \%-3 \mathrm{~mm}$ and $98 \%$ with $5 \%-5 \mathrm{~mm}$. The Gamma Index distribution is shown in the third row of Fig. 5 
$4 D$ PET images. In the following sections, the experimental and simulated $4 \mathrm{D}$ PET images referring to the first monitoring day (data 12/01 and simulation 12/01, respectively) will be analysed using the methods reported in Section 4 . The comparison of the two experimental 4D PET images (data 12/01 and data 12/02) has been already reported in [29. In this paper, the two experimental images are re-analysed by means of the methods reported in Section 4 , using different filters and arbitrary parameters with respect to [29. In any case, the comparison of the two measured 4D PET images is only shown as a benchmark to validate the results obtained between the data and the corresponding simulation.

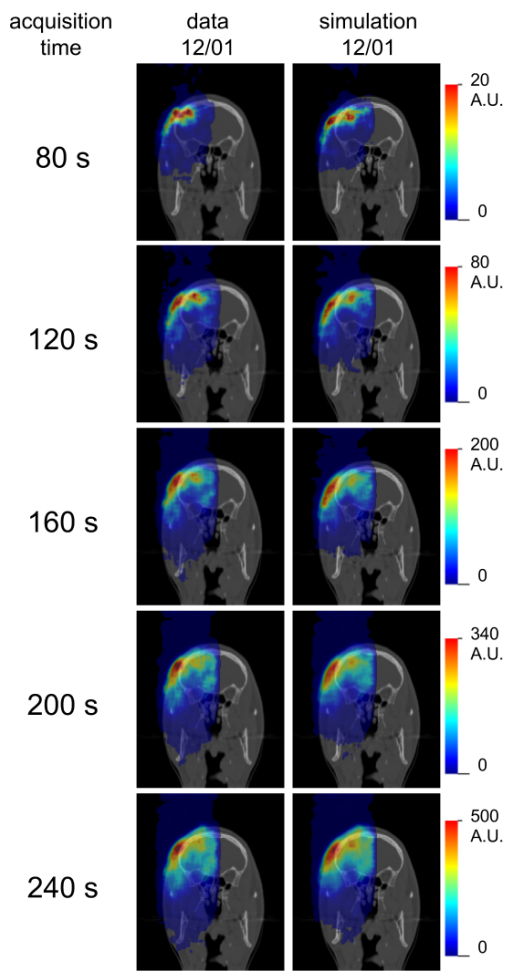

Figure 6: Evolution of the beam-induced activity in time: the same coronal CT slice is shown with the 4D PET images overlaid at different times $(80 s, 120 s, 160 s, 200 s, 240 s$ from the beginning of the treatment delivery). The intensity of the images refers to different scales because of the different time-interval statistics.

In Fig. 6 the evolution of the 4D PET images in time is shown. Each column refers to a different $4 \mathrm{D}$ PET image series and each row to a different acquisition time (every $40 s$ from $80 s$ until the treatment ends at $240 s$ ). The same coronal $\mathrm{CT}$ slice is chosen to show the corresponding activity image slice overlaid in colours. An animation, available in the online version of this article, shows the complete evolution of the measured and simulated images through all the treatment, with a step of $10 \mathrm{~s}$. As stated before, the spatial distribution of the beam-induced activity inside the patient is not straightforwardly correlated 
with the dose distribution (Fig. 5).

As already shown in Fig. 44 the production of positron emitters during the irradiation leads to an increased statistics in the PET image and a spatial expansion in the activity distribution along the beam direction, driven by the increasing protons energy during the treatment delivery, typical for the CNAO synchrotron.

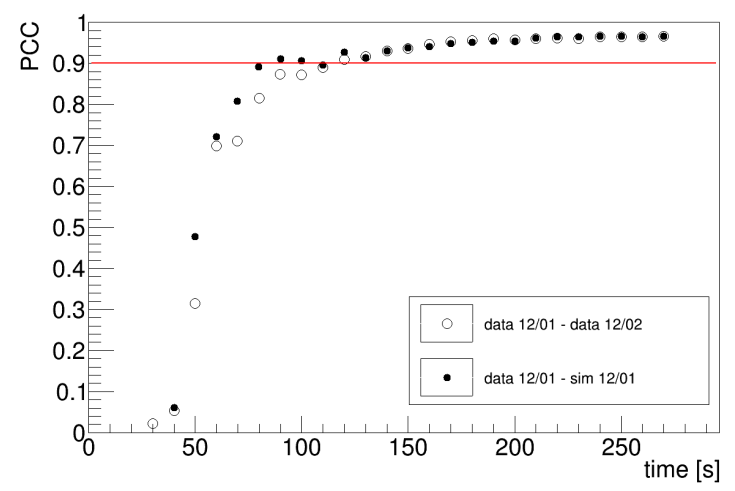

Figure 7: Pearson's Correlation Coefficient analysis in time: the black points represent the agreement between the data and simulation of the first day, while the white points the agreement between the two experimental acquisition. Red line is the 0.9 significant threshold.

Pearson's Correlation Coefficient. As reported in Section 4, the PCC between the experimental and simulated 4D PET images is calculated. The PCC cannot lead to a straightforward agreement evaluation because it is not enough sensitive to range differences, but it can be useful to evaluate the time necessary to collect enough statistics in the PET images for reliable results with further analyses (i.e., BEV and OV methods).

In Fig 7 the PCC evolution as a function of time is shown. The PCC calculated by comparing the experimental and simulated images of the first acquisition day (data 12/01 - sim 12/01 in black points) has the same trend of the PCC obtained considering the two consecutive experimental acquisition (data 12/01 - data 12/02 in white points). In the first $50 \mathrm{~s}$ of irradiation the PET image statistics is not sufficient to define a significant ROI for PCC calculation. After about $120 \mathrm{~s}$ (i.e., half treatment delivery), the PCC reaches values above 0.9 (red line in Fig. 7) and so the two 4D PET images are very similar and can lead to reliable results with the range difference detection analyses.

Beam's Eye View analysis. For the comparison of the experimental and simulated 4D PET images, the distribution of the activity range differences $(\delta R=$ $R_{\text {exp }}-R_{\text {sim }}$ ) were taken into account and the average value and the FWHM of this distribution is monitored in time. Results are summarized in Fig. 8 
A)

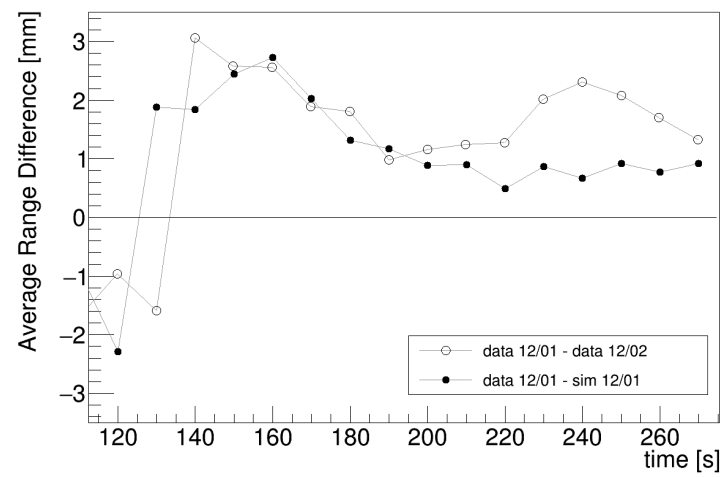

B)

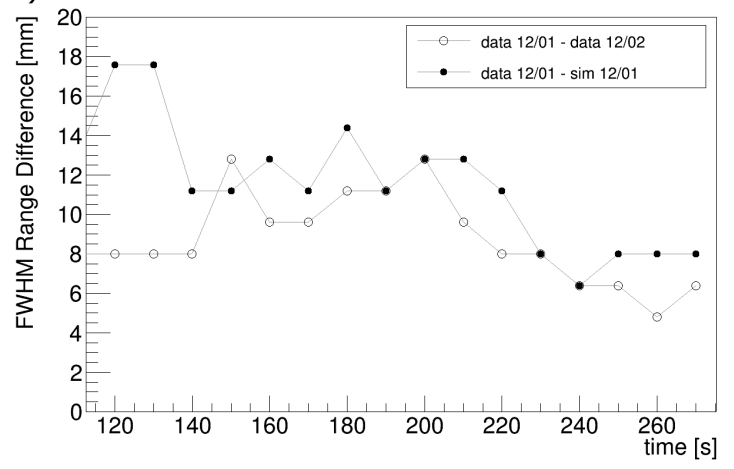

Figure 8: Beam's Eye View analysis in time: A) the average activity range difference; B) the FWHM of the activity range distribution. Black points represent the agreement between data and simulation of the first day, while white points the agreement between the two experimental acquisition. Values before $120 \mathrm{~s}$ from the beginning of the treatment delivery are excluded by the PCC analysis.

The metrics used for the evaluation of the agreement between the two $4 \mathrm{D}$ PET images have almost the same behaviour in the data 12/01 - sim 12/01 and data 12/01 - data 12/02 comparison.

The average activity range difference is less than $3 \mathrm{~mm}$ in absolute value, which is compatible with the uncertainty in the positioning with the laser system available in the CNAO treatment room. The FWHM of the activity range difference distribution is quite large (about $6-8 \mathrm{~mm}$ at the end of acquisition) and reflects the higher sensitivity of the BEV method for possible differences in the positioning of the INSIDE in-beam PET system in the two consecutive days.

Overall View analysis. The mobile support of the INSIDE in-beam PET scanner lacks of a system able to guarantee a reliable and repeatable positioning with respect to the isocenter with an accuracy better than few millimetres. To address this issue, the OV analysis was implemented to reduce the contribution of any 
A)

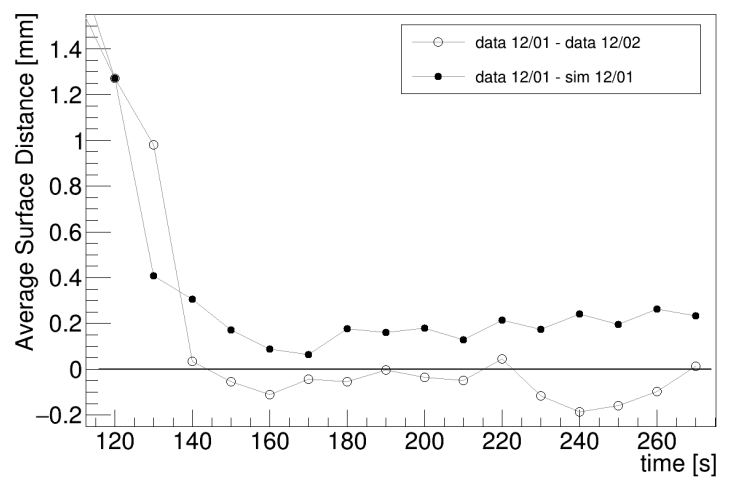

B)

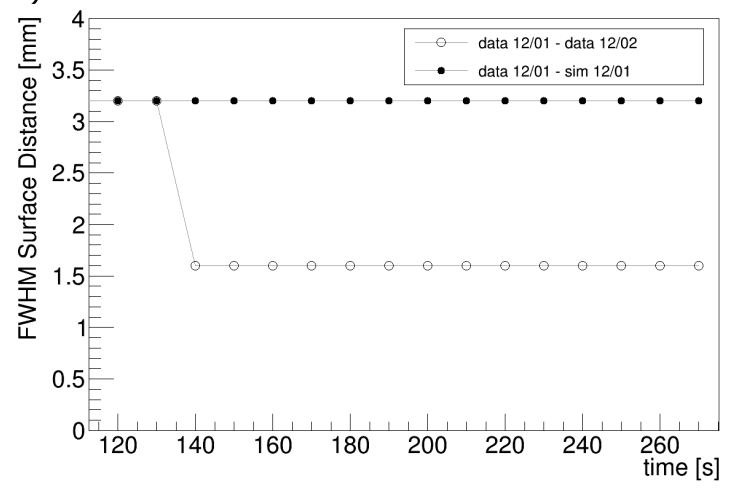

Figure 9: Overall View analysis in time: A) the average minimum distance between the two iso-activity surfaces; B) the FWHM of the iso-activity surface distance distribution. Black points represent the agreement between data and simulation of the first day, while white points the agreement between the two experimental acquisition. Values before $120 \mathrm{~s}$ from the beginning of the treatment delivery are excluded by the PCC analysis.

potential mis-positioning and therefore estimate the performance of the system in case of a dedicated mechanical upgrade to assure reproducible measurement settings. Results are summarized in Fig. 9 .

As shown for the BEV analysis, the metrics chosen for the agreement evaluation exhibits the same trend when comparing the two experimental 4D PET images and the data of the first day with the corresponding simulation. In particular, the average distance between the iso-activity surfaces is less than $1 \mathrm{~mm}$ after $130 \mathrm{~s}$ from the beginning of the treatment delivery and the FWHM is compatible with one or twice the dimension of the voxel $\left(1.6 \times 1.6 \times 1.6 \mathrm{~mm}^{3}\right)$ which is the spatial resolution of the PET images. As expected, the results obtained with the OV analysis reached very quickly an average value compatible with zero and a FWHM compatible with the system intrinsic image resolution because the contribution of mis-positionings or local contour fluctuations is less important with respect to the BEV method. 


\section{Discussion}

In this paper, the treatment of the first patient monitored with the INSIDE in-beam PET scanner is reported, with particular attention to the comparison between the 4D measured activity and the MC simulation.

An important effort was dedicated in the developing of a FLUKA-based MC tool able to model in detail all the features of the INSIDE detector and CNAO beam delivery that can strongly impact on the 4D activity image characteristics.

The use of MC simulation to compare the measured activity with the prescription is standard practice, and has already been done by other groups in the past. These studies performed in-room (e.g., [38]) or after-treatment (e.g., [37) PET, while the INSIDE scanner acquired data during the treatment. In addition, the on-the-fly reconstruction of the activity images, performed with $6 \mathrm{~s}$ delay with respect to the beam delivery, provides range monitoring information to the physicians almost immediately. To our knowledge, a temporal analysis of the activity distribution during patient treatment was performed for the first time by the INSIDE in-beam PET system.

To evaluate the effectiveness and sensitivity of the INSIDE in-beam PET MC simulation tool, the results reported in Section 6 can be compared with literature.

In 38,9 patients were treated with passive scanned proton beams for pathologies in the head and neck region. Immediately after the treatment, a PET examination with an in-room scanner was performed. The time necessary to move the patient inside the full-ring PET system was about 2.3 minutes. The co-registration precision of the acquired PET image to the CT scan was estimated of about $2 \mathrm{~mm}$. By means of an activity profile analysis covering all the irradiated volume, an absolute average activity range difference lower than $5 \mathrm{~mm}$ was found between data and simulation. This result was obtained with a 5 minutes long PET exam and did not change by increasing the acquisition time up to 20 minutes. The root-mean-square deviation of the activity range difference distribution was in the $[4,11] \mathrm{mm}$ interval and did not change depending on the PET acquisition time.

In 37] 20 patients with primary glioma were selected (10 treated with proton boost and 10 with carbon boost in addition to standard treatment). After two fractions, each patient underwent a PET/CT examination with a full-ring commercial scanner (Siemens Biograph mCT) to verify the compliance of the irradiated with the prescribed treatment. The PET acquisition started with a delay of about 5 minutes and lasted 30 minutes, in agreement with the MIRANDA protocol [15]. The 3D PET images were analysed by extracting an activity profile along the beam direction and determining the activity range by applying a robust threshold method reported in [40. The analysis was repeated so as to cover all the irradiated volume. For proton treatments, the comparison between the measured and simulated 3D activity images led to an absolute average activity range difference in the interval $[0.30,7.23] \mathrm{mm}$ (average value among 20 data-simulation comparisons of $4.2 \mathrm{~mm}$ ) with a FWHM of the corresponding distributions in the $[0.50,6.33] \mathrm{mm}$ range. Better results were found 
comparing two experimental measurements (average activity range difference less than $2 \mathrm{~mm}$ with a FWHM of about $1-2 \mathrm{~mm}$ ).

The results obtained by the INSIDE in-beam PET and the comparison between the data with the dedicated FLUKA simulation are compatible with the studies found in literature, but could also be improved by including a precise and reliable positioning system.

In [37. some considerations about the degree of agreement needed to certify the compliance between the delivered and prescribed treatment were attempted, giving as example some patients in which a re-planning was necessary. In 2018, the INSIDE in-beam PET system will be tested in a longitudinal clinical trial to monitor different pathologies in the head and neck region that could benefit most from this monitoring system (e.g., adenoid cystic carcinoma), in order to estimate the sensitivity of this evaluation. Moreover, in the longitudinal clinical trial some pathologies in which functional or morphological changes are not commonly found during the therapeutic period (e.g., meningioma) will also be included in order to verify the specificity of this approach.

\section{Conclusion}

Thanks to state-of-the-art technology, the INSIDE in-beam PET system is able to acquire data in-beam (i.e., during the beam delivery) and analyse data on-the-fly so as to give, with minimum delay, an evaluation of the agreement between the ongoing treatment and the prescription.

In the near future a new mechanical support will be adopted which will remove the limitation of the current setup to only monitor certain treatment configurations in order to cover as many patients as possible; furthermore a dedicated positioning system will guarantee the accuracy and repeatability of the detector position.

The INSIDE in-beam PET MC simulation tool was developed in order to obtain a reliable reference image to be used in the online assessment of the treatment accuracy in hadrontherapy.

The results from the first patient monitored in December 2016 at the CNAO synchrotron facility are very satisfactory. The MC simulation could provide a 4D PET reference image accurate enough to be used in the upcoming wider invivo validation of the INSIDE in-beam PET system. The quantitative analyses implemented to detect activity range differences during the treatment delivery show that the simulated image can successfully replace another experimental acquisition in the same condition of the treatment to be monitored (i.e. two consecutive days acquisition in which the patient's morphology does not change). We strongly believed that all these results are highly significant towards the implementation of adaptive hadrontherapy.

\section{Acknowledgment}

This work was mainly supported by the Italian Ministry of University and Research (MIUR) under the program PRIN 2010-2011 - INSIDE project nr. 
2010P98A75. The work was also partially supported by the CNAO Foundation under the project INSIDE2; EU PF7 project TRIMAGE (grant agreement nr. 602621); Giovanni Goria and CRT Foundations (Bando dei Talenti della Società 2016-2017).

\section{References}

[1] Durante M, Orecchia R, Loeffler JS. Charged-particle therapy in cancer: clinical uses and future perspectives. Nature Reviews Clinical Oncology 2017;

[2] Resch A, Landry G, Kamp F, Cabal G, Belka C, Wilkens J, et al. Quantification of the uncertainties of a biological model and their impact on variable rbe proton treatment plan optimization. Physica Medica: European Journal of Medical Physics 2017;36:91-102.

[3] Bellinzona V, Ciocca M, Embriaco A, Fontana A, Mairani A, Mori M, et al. On the parametrization of lateral dose profiles in proton radiation therapy. Physica Medica: European Journal of Medical Physics 2015;31(5):484-92.

[4] Parodi K. Heavy ion radiography and tomography. Physica Medica: European Journal of Medical Physics 2014;30(5):539-43.

[5] Paganetti H. Range uncertainties in proton therapy and the role of monte carlo simulations. Physics in medicine and biology 2012;57(11):R99.

[6] McGowan S, Burnet N, Lomax A. Treatment planning optimisation in proton therapy. The British journal of radiology 2013;86(1021):20120288-.

[7] Thomas SJ. Margins for treatment planning of proton therapy. Physics in medicine and biology 2006;51(6):1491.

[8] Parodi K. On-and off-line monitoring of ion beam treatment. Nuclear Instruments and Methods in Physics Research Section A: Accelerators, Spectrometers, Detectors and Associated Equipment 2016;809:113-9.

[9] Knopf AC, Lomax A. In vivo proton range verification: a review. Physics in medicine and biology 2013;58(15):R131.

[10] Kelleter L, Wrońska A, Besuglow J, Konefał A, Laihem K, Leidner J, et al. Spectroscopic study of prompt-gamma emission for range verification in proton therapy. Physica Medica: European Journal of Medical Physics 2017;34:7-17.

[11] Piersanti L, Bellini F, Bini F, Collamati F, De Lucia E, Durante M, et al. Measurement of charged particle yields from pmma irradiated by a 220 mev/u 12c beam. Physics in Medicine \& Biology 2014;59(7):1857. 
[12] Dendooven P, Buitenhuis H, Diblen F, Heeres P, Biegun A, Fiedler F, et al. Short-lived positron emitters in beam-on pet imaging during proton therapy. Physics in medicine and biology 2015;60(23):8923.

[13] Del Guerra A, Belcari N, Bisogni M. Positron emission tomography: its 65 years. Riv Nuovo Cimento 2016;39:155.

[14] Zhu X, El Fakhri G. Proton therapy verification with pet imaging. Theranostics 2013;3(10):731.

[15] Combs SE, Bauer J, Unholtz D, Kurz C, Welzel T, Habermehl D, et al. Monitoring of patients treated with particle therapy using positron-emission-tomography (pet): the miranda study. BMC cancer 2012;12(1):133.

[16] Zhu X, España S, Daartz J, Liebsch N, Ouyang J, Paganetti H, et al. Monitoring proton radiation therapy with in-room pet imaging. Physics in medicine and biology 2011;56(13):4041.

[17] Enghardt W, Crespo P, Fiedler F, Hinz R, Parodi K, Pawelke J, et al. Charged hadron tumour therapy monitoring by means of pet. Nuclear Instruments and Methods in Physics Research Section A: Accelerators, Spectrometers, Detectors and Associated Equipment 2004;525(1):284-8.

[18] Sportelli G, Belcari N, Camarlinghi N, Cirrone G, Cuttone G, Ferretti S, et al. First full-beam pet acquisitions in proton therapy with a modular dual-head dedicated system. Physics in medicine and biology 2013;59(1):43.

[19] Camarlinghi N, Sportelli G, Battistoni G, Belcari N, Cecchetti M, Cirrone $\mathrm{G}$, et al. An in-beam pet system for monitoring ion-beam therapy: test on phantoms using clinical 62 mev protons. Journal of Instrumentation 2014;9(04):C04005.

[20] Kraan A, Battistoni G, Belcari N, Camarlinghi N, Cirrone G, Cuttone G, et al. Proton range monitoring with in-beam pet: Monte carlo activity predictions and comparison with cyclotron data. Physica Medica: European Journal of Medical Physics 2014;30(5):559-69.

[21] Bisogni MG, Attili A, Battistoni G, Belcari N, Cerello P, Coli S, et al. Inside in-beam positron emission tomography system for particle range monitoring in hadrontherapy. Journal of medical imaging 2017;4(1):011005-.

[22] Marafini M, Frallicciardi P, Faccini R, Morone C, Voena C, Patera V, et al. The inside project: Innovative solutions for in-beam dosimetry in hadrontherapy. Acta Physica Polonica, A 2015;127(5).

[23] Pennazio F, Attili A, Cerello P, Coli S, Giraudo G, Rivetti A, et al. A study of monitoring performances with the inside system. Acta Physica Polonica, A 2015;127(5). 
[24] Fiorina E, on behalf of the INSIDE Collaboration. An integrated system for the online monitoring of particle therapy treatment accuracy. Nuclear Instruments and Methods in Physics Research Section A: Accelerators, Spectrometers, Detectors and Associated Equipment 2016;824:198-201.

[25] Traini G, Battistoni G, Bollella A, Collamati F, De Lucia E, Faccini R, et al. Design of a new tracking device for on-line beam range monitor in carbon therapy. Physica Medica 2017;34:18-27.

[26] Piliero M, Pennazio F, Bisogni M, Camarlinghi N, Cerello P, Del Guerra A, et al. Full-beam performances of a pet detector with synchrotron therapeutic proton beams. Physics in medicine and biology 2016;61(23):N650.

[27] Piliero M, Belcari N, Bisogni M, Camarlinghi N, Cerello P, Coli S, et al. First results of the inside in-beam pet scanner for the on-line monitoring of particle therapy treatments. Journal of Instrumentation 2016;11(12):C12011.

[28] Ferrero V. The inside project: in-beam pet scanner system features and characterization. Journal of Instrumentation 2017;12(03):C03051.

[29] Ferrero V, Fiorina E, Morrocchi M, Pennazio F, Baroni G, Battistoni $\mathrm{G}$, et al. Online proton therapy monitoring: clinical test of a siliconphotodetector-based in-beam pet. Scientific Reports 2018;8(1):4100.

[30] Rolo M, Bugalho R, Goncalves F, Mazza G, Rivetti A, Silva J, et al. Tofpet asic for pet applications. Journal of Instrumentation 2013;8(02):C02050.

[31] Paganetti H. Proton therapy physics. CRC Press; 2016.

[32] Seco J, Verhaegen F. Monte Carlo techniques in radiation therapy. CRC press; 2013.

[33] Kraan AC. Range verification methods in particle therapy: underlying physics and monte carlo modeling. Frontiers in oncology 2015;5.

[34] Toppi M, Abou-Haidar Z, Agodi C, Alvarez M, Aumann T, Balestra F, et al. Measurement of fragmentation cross sections of c 12 ions on a thin gold target with the first apparatus. Physical Review C 2016;93(6):064601.

[35] Patera V, De Lellis G, Pastrone N, Cerello P, Sanelli C, Mattei I, et al. The foot (fragmentation of target) experiment. PoS 2017;:128.

[36] Linz U. Ion Beam Therapy: Fundamentals, Technology, Clinical Applications. Springer Science \& Business Media; 2011.

[37] Nischwitz SP, Bauer J, Welzel T, Rief H, Jäkel O, Haberer T, et al. Clinical implementation and range evaluation of in vivo pet dosimetry for particle irradiation in patients with primary glioma. Radiotherapy and Oncology 2015;115(2):179-85. 
[38] Min CH, Zhu X, Winey BA, Grogg K, Testa M, El Fakhri G, et al. Clinical application of in-room positron emission tomography for in vivo treatment monitoring in proton radiation therapy. International Journal of Radiation Oncology* Biology* Physics 2013;86(1):183-9.

[39] Bauer J, Unholtz D, Sommerer F, Kurz C, Haberer T, Herfarth K, et al. Implementation and initial clinical experience of offline pet/ct-based verification of scanned carbon ion treatment. Radiotherapy and Oncology 2013;107(2):218-26.

[40] Frey K, Unholtz D, Bauer J, Debus J, Min C, Bortfeld T, et al. Automation and uncertainty analysis of a method for in-vivo range verification in particle therapy. Physics in medicine and biology 2014;59(19):5903.

[41] Battistoni G, Bauer J, Boehlen TT, Cerutti F, Chin MP, Augusto RDS, et al. The fluka code: an accurate simulation tool for particle therapy. Frontiers in oncology $2016 ; 6$.

[42] Ferrari A, Sala PR, Fasso A, Ranft J. Fluka: A multi-particle transport code (program version 2005). Tech. Rep.; 2005.

[43] Böhlen T, Cerutti F, Chin M, Fassò A, Ferrari A, Ortega P, et al. The fluka code: developments and challenges for high energy and medical applications. Nuclear Data Sheets 2014;120:211-4.

[44] Vlachoudis V, et al. Flair: a powerful but user friendly graphical interface for fluka. In: Proc. Int. Conf. on Mathematics, Computational Methods \& Reactor Physics (M\&C 2009), Saratoga Springs, New York. 2009,.

[45] Lima TV, Dosanjh M, Ferrari A, Molineli S, Ciocca M, Mairani A. Monte carlo calculations supporting patient plan verification in proton therapy. Frontiers in oncology 2016;6.

[46] Molinelli S, Mairani A, Mirandola A, Freixas GV, Tessonnier T, Giordanengo S, et al. Dosimetric accuracy assessment of a treatment plan verification system for scanned proton beam radiotherapy: one-year experimental results and monte carlo analysis of the involved uncertainties. Physics in medicine and biology 2013;58(11):3837.

[47] Giordanengo S, Garella MA, Marchetto F, Bourhaleb F, Ciocca M, Mirandola A, et al. The cnao dose delivery system for modulated scanning ion beam radiotherapy. Medical Physics 2015;42(1):263-75.

[48] Mirandola A, Molinelli S, Vilches Freixas G, Mairani A, Gallio E, Panizza $\mathrm{D}$, et al. Dosimetric commissioning and quality assurance of scanned ion beams at the italian national center for oncological hadrontherapy. Medical Physics 2015;42(9):5287-300. 
[49] Parodi K, Mairani A, Sommerer F. Monte carlo-based parametrization of the lateral dose spread for clinical treatment planning of scanned proton and carbon ion beams. Journal of Radiation Research 2013;54:i91-6.

[50] Schneider W, Bortfeld T, Schlegel W. Correlation between ct numbers and tissue parameters needed for monte carlo simulations of clinical dose distributions. Physics in medicine and biology 2000;45(2):459.

[51] Rossi S. The status of cnao. The European Physical Journal Plus 2011;126(8):1-39.

[52] Pullia MG. Synchrotrons for hadrontherapy. Reviews of Accelerator Science and Technology 2009;2(01):157-78.

[53] Pennazio F, Bisogni M, Camarlinghi N, Cerello P, Fiorina E, Morrocchi $\mathrm{M}$, et al. $\mathrm{Pv}-0562$ : Hadron-therapy monitoring with in-beam pet: measurements and simulations of the inside pet scanner. Radiotherapy and Oncology 2016;119:S270.

[54] Stark JA. Adaptive image contrast enhancement using generalizations of histogram equalization. IEEE Transactions on image processing 2000;9(5):889-96.

[55] Kuess P, Birkfellner W, Enghardt W, Helmbrecht S, Fiedler F, Georg D. Using statistical measures for automated comparison of in-beam pet data. Medical Physics 2012;39(10):5874-81.

[56] Nishio T, Miyatake A, Ogino T, Nakagawa K, Saijo N, Esumi H. The development and clinical use of a beam on-line pet system mounted on a rotating gantry port in proton therapy. International Journal of Radiation Oncology* Biology* Physics 2010;76(1):277-86.

[57] Low DA, Harms WB, Mutic S, Purdy JA. A technique for the quantitative evaluation of dose distributions. Medical physics 1998;25(5):656-61. 